Original Article

\title{
Prospective Study on the Prevalence of Secondary Hypertension among Hypertensive Patients Visiting a General Outpatient Clinic in Japan
}

\author{
Masao OMURA, Jun SAITO, Kunio YAMAGUCHI* , Yukio KAKUTA**, and Tetsuo NISHIKAWA
}

\begin{abstract}
Secondary hypertension (SH) including endocrine hypertension has been reported to be uncommon. We estimated the prevalence of $\mathrm{SH}$ among hypertensive patients. We prospectively studied 1,020 hypertensive patients. As an initial screening, we measured plasma aldosterone concentration, plasma renin activity, serum cortisol concentration and plasma catecholamine concentration and conducted abdominal ultrasonography (US). As a secondary screening, we performed furosemide plus upright test, captopril renography, dexamethasone suppression test, 24-h urine catecholamine measurement and abdominal CT. Finally, primary aldosteronism with the exception of idiopathic hyperaldosteronism, pheochromocytoma, and Cushing's syndrome were diagnosed by histopathological examination of surgical specimens. Idiopathic hyperaldosteronism was clinically diagnosed by adrenocorticotrophic hormone (ACTH)-stimulated adrenal venous sampling and renovascular hypertension by renal arteriography. There were 61 patients with primary aldosteronism, 5 with renovascular hypertension, 11 with Cushing's syndrome, 10 with preclinical Cushing's syndrome and 6 with pheochromocytoma, and the prevalence of $\mathrm{SH}$ was $9.1 \%$ among 1,020 hypertensive patients. In 76 $(82 \%)$ of 93 patients with $\mathrm{SH}$, hypertension was cured or improved after unilateral adrenalectomy, transsphenoidal pituitary adenectomy or percutaneous transluminal angioplasty. With the exception of US and CT, all initial and secondary screening tests were found to be sensitive and specific for differentiating SH from essential hypertension (EH). In conclusion, the measurement of various hormone concentrations was very sensitive for ruling out SH-a condition for which, in the present study, there were few specific signs or symptoms-while CT and US examinations were not always useful for differentiating SH from EH. The prevalence of curable SH among hypertensive subjects was higher in this study, which was conducted by our simple method of screening tests, than in previous reports. Hypertensive patients should be screened for $\mathrm{SH}$ and the underlying disease treated appropriately to avoid long-term use of antihypertensive drugs and risks of atherosclerotic complications. (Hypertens Res 2004; 27: 193-202)
\end{abstract}

Key Words: secondary hypertension, primary aldosteronism, Cushing's syndrome, pheochromocytoma, renovascular hypertension

\section{Introduction}

The prevalence of hypertension has been well documented in epidemiological studies conducted in many countries $(1,2)$. The proportion of patients with curable secondary hypertension (SH), including patients with primary aldosteronism
(PA), renovascular hypertension (RVH), Cushing's syndrome and pheochromocytoma $(\mathrm{Ph})$, to the total hypertensive population has been reported to be from 0.1 to $1.2 \%$ (3-5). The prevalence of PA, one of the common forms of $\mathrm{SH}$, has been reported to range from 4.6 to $9.5 \%$ among hypertensive patients $(6-11)$ since it was first investigated by Gordon et al. in 1994 (6). On the other hand, to our knowl-

From the Department of Medicine, ${ }^{*}$ Department of Urology, and ${ }^{* *}$ Department of Pathology, Yokohama Rosai Hospital, Yokohama, Japan. Address for Reprints: Masao Omura, M.D., Division of Endocrinology and Metabolism, Department of Internal Medicine, Social Insurance Central General Hospital, 3-22-1 Hyakunin-cho, Shinjuku-ku, Tokyo 169-0073, Japan. E-mail: omura_shahochu@yahoo.co.jp

Received August 4, 2003; Accepted in revised form December 17, 2003. 
Table 1. Concentrations of Plasma Aldosterone, Cortisol, Adrenaline, Noradrenaline and Dopamine, and Plasma Renin Activity in 22 Hypertensives before and 2 Weeks after Administration of Budralazine

\begin{tabular}{lccc}
\hline & Before administration of budralazine & 2 weeks after administration of budralazine & $p$ value \\
\hline Aldosterone $(\mathrm{ng} / \mathrm{dl})$ & $13.7 \pm 7.5$ & $14.2 \pm 8.3$ & 0.59 \\
Cortisol $(\mu \mathrm{g} / \mathrm{dl})$ & $11.5 \pm 5.1$ & $11.2 \pm 4.3$ & 0.23 \\
Adrenaline $(\mathrm{ng} / \mathrm{ml})$ & $0.08 \pm 0.04$ & $0.05 \pm 0.04$ & 0.23 \\
Noradrenalin $(\mathrm{ng} / \mathrm{ml})$ & $0.43 \pm 0.19$ & $0.36 \pm 0.23$ & 0.06 \\
Dopamine $(\mathrm{ng} / \mathrm{ml})$ & $0.03 \pm 0.02$ & $0.03 \pm 0.05$ & 0.60 \\
Plasma renin activity $(\mathrm{ng} / \mathrm{ml} / \mathrm{h})$ & $0.6 \pm 0.4$ & $0.6 \pm 0.4$ & 0.77 \\
\hline
\end{tabular}

Number of patients with: aldosterone-producing adenoma, 13; idiopathic hyperaldosteronism, 2; Cushing's syndrome, 3; essential hypertension, 4. All data are shown as the means \pm SEM. Student's $t$-test was used to compare changes in each parameter between the pre- and post-treatment stages. Values of $p<0.05$ were considered to indicate statistical significance.

edge there have been no recent reports describing the prevalence of SH other than the paper reported by Anderson et al. in 1994 (12). One of the reasons for this is that relatively extensive investigations are necessary to identify secondary forms of hypertension with reasonable certainty.

Therefore, we examined 1,020 hypertensive patients for $\mathrm{SH}$ at the outpatient clinic in Yokohama Rosai Hospital from 1995 to 1999 . We attempted to design a simple screening scheme for $\mathrm{SH}$ using various hormonal examinations, and evaluated the prevalence of $\mathrm{SH}$ among all hypertensive patients studied.

\section{Methods}

\section{Patient Selection}

We conducted a prospective analysis of 1,020 hypertensive patients at the general outpatient clinic in Yokohama Rosai Hospital from 1995 to 1999 . Hypertensive patients who had been suspected of having endocrinologic diseases and were referred to Yokohama Rosai Hospital for further examinations to differentiate $\mathrm{SH}$ from essential hypertension $(\mathrm{EH})$ were excluded from this study. Informed consent was obtained from all subjects after explanation of the purpose and potential risk of the study. The protocol was approved by the Ethics Committee of Yokohama Rosai Hospital. Hypertension was defined as a blood pressure (BP) above 140/90 $\mathrm{mmHg}$, according to the classification of BP for adults in the Guidelines for the Management of Hypertension for General Practitioners (JSH 2000) (2). All patients underwent a complete physical examination, including auscultation of the heart and lungs, palpation of peripheral pulses and examination of the ocular fundi. Laboratory tests included measurements of hemoglobin, white blood cell count, serum electrolytes, serum creatinine, serum uric acid, blood glucose, serum cholesterol, serum triglycerides, and serum transaminases, and a test for proteinuria and urinary sediment. ECG was recorded in all patients. Based on these results, patients with renal failure, heart failure, and acute-phase coronary heart disease or cerebrovascular disease were excluded from this investigation. No patients were taking any antihypertensive drugs at the time of the initial screening. In some patients, in whom withdrawal of the antihypertensive treatment seemed unsafe, budralazine was allowed for 2 weeks before initial screening, because this drug does not significantly alter plasma aldosterone concentration (PAC), plasma renin activity (PRA), serum cortisol concentration $(F)$ or plasma catecholamine concentrations (PCA), as shown in Table 1.

\section{Primary Screening Tests}

At the primary screening, blood samples were collected in the morning after the patient had rested in a supine position for $30 \mathrm{~min}$, and PAC, $F$ and PRA were measured by radioimmunoassay using commercially available kits. PCA and 24-h urine catecholamines (UCA) were measured by high performance liquid chromatography. All hypertensive patients also underwent abdominal ultrasonography (US).

\section{Secondary Screening Tests}

\section{Furosemide Plus Upright Test}

The furosemide plus upright test was performed in the morning after resting for at least $30 \mathrm{~min}$ in the supine position, as previously reported (13). Blood samples for estimating PAC and PRA were obtained $120 \mathrm{~min}$ after intravenous injection of $40 \mathrm{mg}$ furosemide with the subject standing upright for the full $120 \mathrm{~min}$ before sampling.

\section{Captopril Renography}

Captopril renography was performed by using ${ }^{99 \mathrm{~m}}$ Tc-diethylenetriaminepentaacetic acid as a tracer $60 \mathrm{~min}$ after oral administration of $25 \mathrm{mg}$ captopril according to the method described previously by Helin et al. (14).

\section{Overnight Dexamethasone Suppression Test}

One milligram of dexamethasone was orally administered at 23:00, and blood for determination of $F$ was withdrawn at 09:00 the next morning (15). 
Measurement of UCA

Twenty-four-hour urine catecholamines were measured by high performance liquid chromatography.

Abdominal Computed Tomography

We obtained CT images of adrenal glands by performing thin-section scans with a 2-mm collimation, at 2-mm intervals after intravenous injection of contrast medium. The CT scanning was done under conditions of $120 \mathrm{kVp}, 100 \mathrm{~mA}$ and 30-s scanning time. The scans were reconstructed with a standard reconstruction algorithm.

\section{Criteria for Suspected Secondary Hypertension after Initial and Second Screening}

Patients with a PRA $<1.0 \mathrm{ng} / \mathrm{ml} / \mathrm{h}$ and a PAC $>12.0 \mathrm{ng} / \mathrm{dl}$ were diagnosed as having hyporeninemic hyperaldosteronemia and underwent a furosemide plus upright test. Patients with a PRA $>3.0 \mathrm{ng} / \mathrm{ml} / \mathrm{h}$ and a PAC $>12.0 \mathrm{ng} / \mathrm{dl}$ were diagnosed as having hyperreninemic hyperaldosteronemia and underwent captopril renography. Patients with an $F>20$ $\mu \mathrm{g} / \mathrm{dl}$ were diagnosed as having hypercortisolemia and they underwent an overnight dexamethasone suppression test. Patients with a PCA more than three times the normal upper limit were diagnosed as having hypercatecholaminemia, and UCA was measured in these patients. Patients with abnormal US findings of adrenal glands were suspected of adrenal diseases. They were further examined by a furosemide plus upright test, an overnight dexamethasone suppression test and UCA measurement. A CT scan of the adrenal glands was also performed in all patients with abnormal findings in the primary screening tests, i.e., PRA, PAC, F, PCA, and US.

\section{Final Diagnosis and Treatment of Diseases of Sec- ondary Hypertension}

\section{Primary Aldosteronism}

Patients with a PRA $<1.0 \mathrm{ng} / \mathrm{ml} / \mathrm{h}$ after intravenous administration of $40 \mathrm{mg}$ furosemide followed by $120 \mathrm{~min}$ of standing upright were suspected of PA. PA was further confirmed by a captopril loading test using the criterion of a PAC/PRA ratio of more than 20 at $90 \mathrm{~min}$ after a $50 \mathrm{mg}$ load of captopril (16). Then, patients with PA underwent adrenocorticotrophic hormone (ACTH)-stimulated adrenal venous sampling (AVS) to differentiate unilateral from bilateral aldosterone hypersecretion according to the method reported previously (17). Aldosterone hypersecretion was diagnosed when aldosterone concentrations in the adrenal venous effluents were more than $1,400 \mathrm{ng} / \mathrm{dl} 30 \mathrm{~min}$ after ACTH stimulation. Patients with confirmed bilateral aldosterone hypersecretion were clinically diagnosed as having idiopathic hyperaldosteronism (IHA). Patients with unilateral aldosterone hypersecretion confirmed by ACTH-stimulated AVS underwent unilateral adrenalectomy. Diagnoses of aldosterone producing adenoma (APA), unilateral adrenal hyperplasia
(UAH) (18) and unilateral multiple adrenocortical micronodules (UMN) (17) were finally made by histopathological examination of the resected adrenal glands according to the methods reported previously (17).

Renovascular Hypertension

Patients with hyperreninemic hyperaldosteronemia underwent captopril renography. Prolongation of the time to reach maximal activity $\left(T_{\max }\right)$ and asymmetry in renal handling of isotope were criteria for further screening for RVH (14). Patients with abnormal renograms were admitted to hospital for renal arteriography and other investigations. Arteriographic verification was required for a diagnosis of renal artery stenosis or intrarenal occlusion. Patients diagnosed with RVH were treated with percutaneous transluminal angioplasty (PTA).

\section{Cushing's Syndrome}

Patients showing $F>3.0 \mu \mathrm{g} / \mathrm{dl}$ after oral administration of $1 \mathrm{mg}$ of dexamethasone were suspected of Cushing's syndrome due to adrenocortical adenoma (CS), Cushing's disease (CD) or preclinical Cushing's syndrome due to adrenocortical tumor (PCS). CS and PCS were clinically diagnosed by $\mathrm{CT}$ scans and ${ }^{131} \mathrm{I}$-cholesterol scintigraphy. Six patients

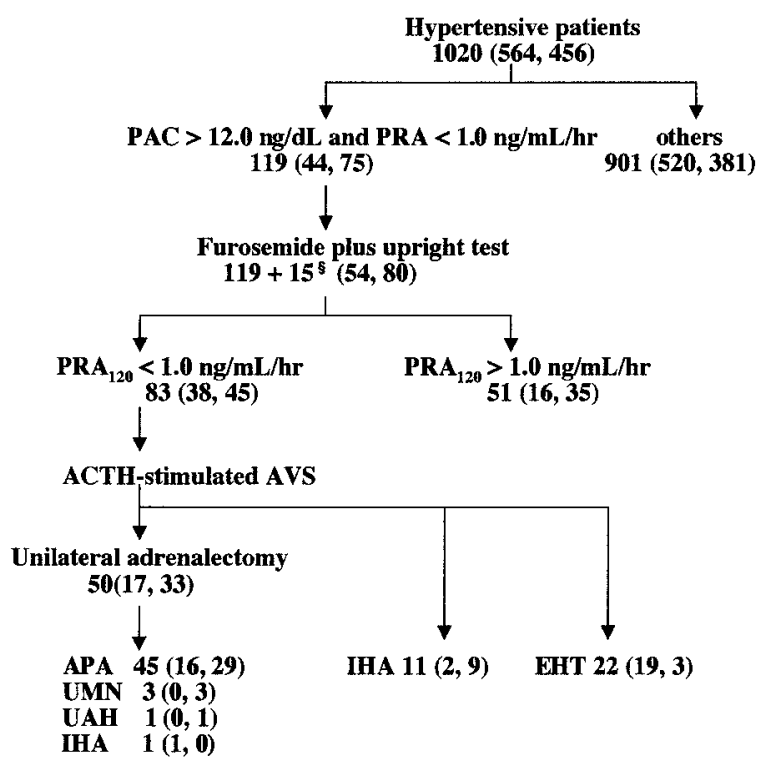

Fig. 1. Sequential analysis for screening primary aldosteronism. PAC, plasma aldosterone concentration; PRA, plasma renin activity; PRA120, PRA 120 min after intravenous administration of $40 \mathrm{mg}$ furosemide and standing upright; AVS, adrenal venous sampling; APA, aldosterone-producing adenoma; UMN, unilateral multiple adrenocortical micronodules; UAH, unilateral adrenal hyperplasia; IHA, idiopathic hyperaldosteronism; EHT, essential hypertension. § Patients with adrenal tumor detected only by US. The numbers of male and female patients are shown in parentheses, respectively. 


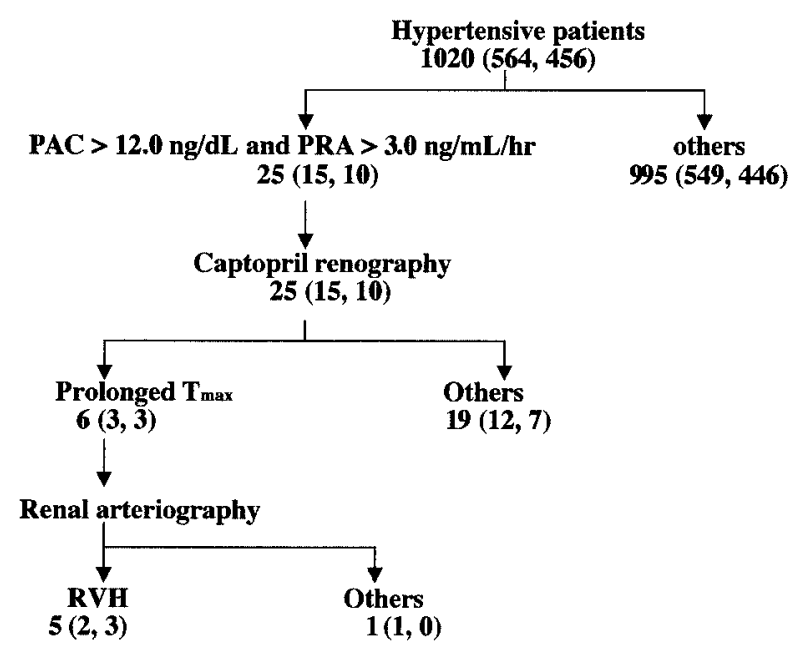

Fig. 2. Sequential analysis for diagnosing renovascular hypertension. PAC, plasma aldosterone concentration; PRA, plasma renin activity; $\mathrm{T}_{\max }$, time to reach maximal activity; $R V H$, renovascular hypertension. The numbers of male and female patients are shown in parentheses, respectively.

with CS and five patients with PCS, all of whom gave informed consent, underwent unilateral adrenalectomy. Diagnoses of CS and PCS were proven by histopathological examination of the resected adrenal tumors. CD was confirmed by pituitary MRI and finally diagnosed by histopathological examinations of resected specimens obtained from transsphenoidal pituitary adenectomy.

\section{Pheochromocytoma}

Diagnosis of $\mathrm{Ph}$ was further confirmed by abdominal MRI and ${ }^{131} \mathrm{I}$-meta-iodobenzylguanidine ( $\left.{ }^{131} \mathrm{I}-\mathrm{MIBG}\right)$ scintigraphy, and the diagnosis was finally proven by histopathological findings of the resected adrenomedullary tumor.

\section{Results}

\section{Screening for Primary Aldosteronism}

As shown in Fig. 1, a furosemide plus upright test was conducted in 119 patients with hyporeninemic hyperaldosteronemia and in another 15 patients with adrenal tumor initially detected by US. Among them, PA was suspected in 83 patients with a PRA $<1.0 \mathrm{ng} / \mathrm{ml} / \mathrm{h} 120 \mathrm{~min}$ after the furosemide plus upright test. US examination could not demonstrate any abnormalities in the adrenals among those patients with a PRA $<1.0 \mathrm{ng} / \mathrm{ml} / \mathrm{h} \quad 120 \mathrm{~min}$ after the furosemide plus upright test. Eleven of 83 patients were clinically diagnosed as having IHA based on bilateral aldosterone hypersecretion revealed by ACTH-stimulated AVS. Fifty patients who showed unilateral hypersecretion of aldosterone underwent unilateral adrenalectomy. Histopathological examinations of the resected adrenal glands confirmed a

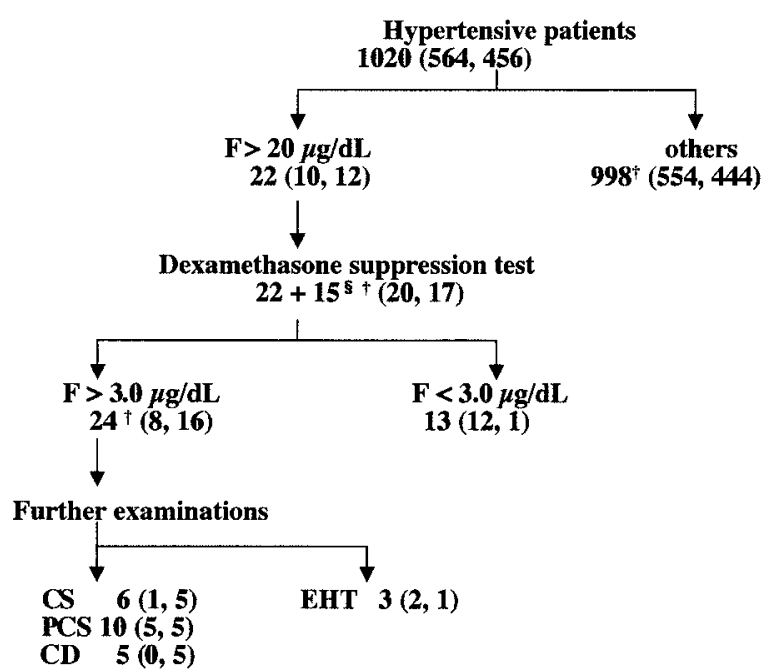

Fig. 3. Sequential analysis for detecting Cushing's syndrome. F, serum cortisol concentration; CS, Cushing's syndrome due to adrenal adenoma; PCS, preclinical Cushing's syndrome; CD, Cushing's disease due to pituitary adenoma; EHT, essential hypertension. § Patients with adrenal tumor detected only by $U S{ }^{\dagger}{ }^{\dagger}$ including 1 patient with $C S$ and 8 patients with PCS with $\mathrm{F}<20 \mu \mathrm{g} / d l$ at the initial screening test. The numbers of male and female patients are shown in parentheses, respectively.

Table 2. Positive and Negative Findings of US Images for Detecting Adrenal Lesions in Patients with APA, UMN, UAH, IHA, CS, PCS, Ph and NF.

\begin{tabular}{lcc}
\hline & \multicolumn{2}{c}{ Number of patients } \\
\cline { 2 - 3 } & $\begin{array}{c}\text { With an adrenal lesion } \\
\text { that could be } \\
\text { detected by US }\end{array}$ & $\begin{array}{c}\text { With an adrenal lesion } \\
\text { that could not be } \\
\text { detected by US }\end{array}$ \\
\hline APA & $18(0)$ & 27 \\
UMN & $0(0)$ & 3 \\
UAH & $0(0)$ & 1 \\
IHA & $0(0)$ & 12 \\
CS & $4(1)$ & 2 \\
PCS & $8(8)$ & 2 \\
Ph & $5(1)$ & 1 \\
NF & $5(5)$ & 0 \\
\hline Total & $40(15)$ & 48
\end{tabular}

Numbers of patients who did not show any abnormalities in the initial screening tests are shown in parenthesis. US, ultrasonography; APA, aldosterone-producing adenoma; UMN, unilateral multiple adrenocortical micronodules; UAH, unilateral adrenal hyperplasia; IHA, idiopathic hyperaldosteronism; CS, Cushing's syndrome due to adrenocortical adenoma; PCS, preclinical Cushing's syndrome; $\mathrm{Ph}$, pheochromocytoma; NF, non-functioning adrenal tumor.

diagnosis of APA in 45 patients, UMN in 3 , UAH in 1 and IHA in 1. 
Table 3. Incidence of Essential and Secondary Hypertension among Hypertensive Patients, in Comparison with Previous Reports

\begin{tabular}{|c|c|c|c|c|c|}
\hline & $\begin{array}{l}\text { Omura et al. } \\
\quad(2003)\end{array}$ & $\begin{array}{l}\text { Anderson et al. (12) } \\
\text { (1994) }\end{array}$ & $\begin{array}{l}\text { Sinclair et al.(5) } \\
\text { (1987) }\end{array}$ & $\begin{array}{l}\text { Danielson et al.(4) } \\
\text { (1981) }\end{array}$ & $\begin{array}{l}\text { Rudnick et al.(3) } \\
\text { (1977) }\end{array}$ \\
\hline Numbers of patient examined & 1,020 & 4,429 & 3,783 & 1,000 & 665 \\
\hline Essential hypertension (\%) & 90.9 & 89.5 & 92.1 & 95.3 & 94.0 \\
\hline Renal hypertension $(\%)$ & $0^{*}$ & 1.8 & 5.6 & 2.4 & 5.0 \\
\hline Primary aldosteronism (\%) & 6.0 & 1.5 & 0.3 & 0.1 & ND \\
\hline Renovascular hypertension (\%) & 0.5 & 3.3 & 0.7 & 1.0 & 0.2 \\
\hline Cushing's syndrome $(\%)$ & 1.0 & 0.6 & 0.1 & 0.1 & 0.2 \\
\hline Preclinical Cushing's syndrome (\%) & 1.0 & ND & ND & ND & ND \\
\hline Pheochromocytoma (\%) & 0.6 & 0.3 & 0.1 & 0.2 & ND \\
\hline Others $(\%)$ & 0 & 0 & 1.0 & 0.8 & 0.4 \\
\hline
\end{tabular}

${ }^{*}$ Patients with renal failure were excluded from this investigation. \%, prevalence of essential and secondary hypertension, such as renal hypertension, primary aldosteronism, and so on, among hypertensive patients examined. ND, not determined.

\section{Screening for Renovascular Hypertension}

As shown in Fig. 2, hyperreninemic hyperaldosteronemia was confirmed in 25 patients, 6 of whom were suspected of having RVH from the findings of captopril renography. Renal arteriography revealed stenosis of the renal arteries in 5 of 6 patients, and they were diagnosed as having RVH. Images of renal arteriography also demonstrated that RVH had possibly been induced in these patients by fibromuscular degeneration and in another 2 patients by atherosclerotic changes.

\section{Screening for Cushing's Syndrome}

As shown in Fig. 3, hypercortisolemia was demonstrated in 22 patients. These 22 patients together with 15 patients with adrenal tumor initially detected by US were examined by a dexamethasone suppression test. Twenty-four patients were suspected of CS, CD or PCS. Further examinations such as abdominal CT scans, ${ }^{131}$ I-cholesterol scintigraphy and pituitary MRI allowed diagnosis of CS in 6 patients, PCS in 10 and $\mathrm{CD}$ in 5 . Among these 21 patients, 8 patients with PCS and 1 patient with CS with an $F<20 \mu \mathrm{g} / \mathrm{dl}$ were detected initially by US (Table 2). All 6 patients clinically diagnosed with CS and 5 of 10 patients diagnosed with PCS underwent unilateral adrenalectomy, and all 5 patients clinically diagnosed with CD underwent transsphenoidal pituitary adenectomy. The final diagnoses of CS, PCS and CD were confirmed by histopathological examinations of the resected specimens.

\section{Screening for Pheochromocytoma}

UCA was measured in 42 patients showing increased PCA and another 15 patients with adrenal tumor initially detected by US. Among the 57 patients, 6 showed elevated UCA while 52, including 1 patient with $\mathrm{Ph}$, were within normal

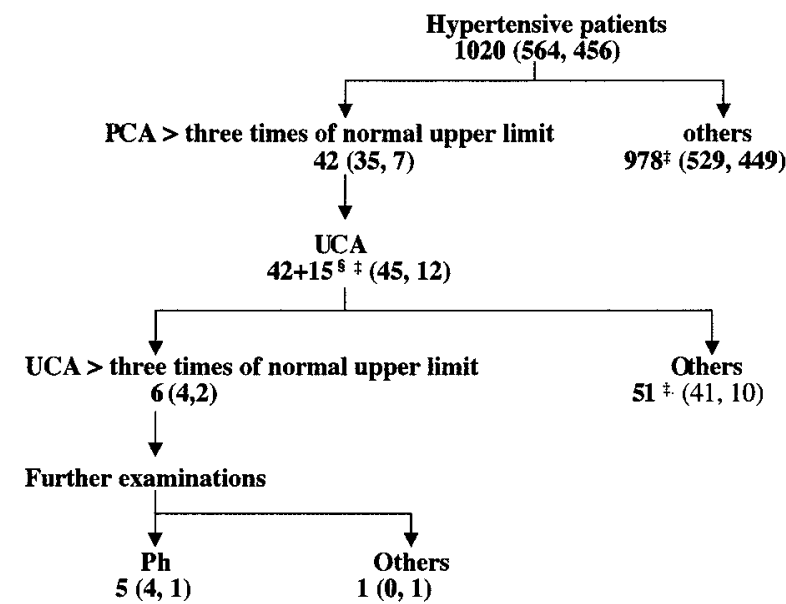

Fig. 4. Sequential analysis for screening pheochromocytoma. PCA, plasma catecholamine concentration; UCA, 24$h$ urine catecholamines; $P$, pheochromocytoma. ${ }^{\S}$ Patients with adrenal tumor detected only by US; $¥$ including one case of Ph. The numbers of male and female patients are shown in parentheses, respectively.

limits. Imaging studies such as abdominal MRI and ${ }^{131}$ I-MIBG scintigraphy revealed adrenomedullary tumors in 5 of 6 patients (Fig. 4). The 5 patients underwent unilateral adrenalectomy. One patient with normal UCA and without abnormalities in any of the primary screening tests except US underwent unilateral adrenalectomy for suspected nonfunctioning adrenal tumor; however, the adrenal tumor was finally diagnosed as $\mathrm{Ph}$ by histopathological examinations of the resected adrenal gland.

\section{Diagnostic Imaging in the Detection of Adrenal Le- sions}

US aided the detection of 40 adrenal lesions in 1,020 hypertensive patients. Among these 40 patients, 1 with CS, 8 with 
Table 4. Sensitivity and Specificity of Each Primary and Secondary Screening Test for Differentiating Secondary Hypertension from Essential Hypertension

\begin{tabular}{lccc}
\hline & Number of patients examined & Sensitivity & Specificity \\
\hline Primary screening test & & & 1.00 \\
Hyporeninemic hyperaldosteronemia & 1,020 & 1.00 & 0.94 \\
Hyperreninemic hyperaldosteronemia & 1,020 & 0.91 & 0.98 \\
Hypercortisolemia & 1,020 & 0.83 & 0.99 \\
Hypercathecholaminemia & 1,020 & 0.42 & 0.96 \\
Abdominal ultrasonography & 1,020 & & 1.00 \\
Secondary screening test & & 1.00 & 0.70 \\
Furosemide plus upright test & 134 & 1.00 & 0.81 \\
Overnight dexamethasone suppression test & 37 & 1.00 & 0.95 \\
Captopril renography & 25 & 0.83 & 0.98 \\
Urinary excretion of catecholamines & 57 & 0.63 & 1.00 \\
Abdominal CT scan & 223 & & \\
\hline
\end{tabular}

The methods and criteria of the primary and secondary screening tests are described in the Methods section.

Table 5. Outcomes 1 Year after the Surgical Treatment for Patients with Each Disorder of Endocrine Hypertension

\begin{tabular}{|c|c|c|c|c|c|}
\hline & \multirow{3}{*}{ Number of patients } & \multicolumn{4}{|c|}{ Number of patients } \\
\hline & & \multirow{2}{*}{$\begin{array}{l}\text { Undergoing } \\
\text { surgical treatment }\end{array}$} & \multicolumn{3}{|c|}{ After surgical treatment } \\
\hline & & & Cured & Improved & Not improved \\
\hline APA & 45 & 45 & 32 & 13 & 0 \\
\hline UMN & 3 & 3 & 3 & 0 & 0 \\
\hline UAH & 1 & 1 & 1 & 0 & 0 \\
\hline IHA & 12 & 1 & 0 & 0 & 1 \\
\hline RVH & 5 & 5 & 3 & 2 & 0 \\
\hline CS & 6 & 6 & 5 & 1 & 0 \\
\hline PCS & 10 & 5 & 4 & 1 & 0 \\
\hline $\mathrm{CD}$ & 5 & 5 & 5 & 0 & 0 \\
\hline $\mathrm{Ph}$ & 6 & 6 & 5 & 1 & 0 \\
\hline Total & 93 & 77 & 58 & 18 & 1 \\
\hline
\end{tabular}

Cured: became completely normotensive without any antihypertensive agents 1 year after the surgical treatment; improved: fairly well controlled and needed lower doses and fewer antihypertensive drug than before the surgical treatment. APA, aldosterone-producing adenoma; UMN, unilateral multiple adrenocortical micronodules; UAH, unilateral adrenal hyperplasia; IHA, idiopathic hyperaldosteronism; RVH, renovascular hypertension; CS, Cushing's syndrome due to adrenocortical adenoma; PCS, preclinical Cushing's syndrome; CD, Cushing's disease; $\mathrm{Ph}$, pheochromocytoma.

$\mathrm{PCS}, 1$ with $\mathrm{Ph}$ and 5 with non-functioning adrenal tumor were suspected of adrenal disorders at the initial US, even though they did not show any abnormalities in the primary screening tests, including basal levels of PAC, PRA, $F$ and PCA. Moreover, hypertension was cured after unilateral adrenalectomy in 1 patient with CS, 4 patients with PCS and 1 patient with $\mathrm{Ph}$ detected initially by US. On the other hand, US did not detect adrenal lesions in 48 patients who were finally diagnosed with APA, UMN, UAH, IHA, CS, PCS and $\mathrm{Ph}$ (Table 2).

CT revealed adrenal lesions in 45 of 224 patients who demonstrated some abnormal findings in the primary screening tests. On the other hand, CT detected no abnormal findings in the adrenal glands of 22 patients with APA, 3 with
UMN and 1 with UAH who had shown hyporeninemic hyperaldosteronemia.

\section{Prevalence of Secondary Hypertension}

The present data demonstrated SH in $93(9.1 \%)$ of 1,020 hypertensive patients. Thus, the prevalences of PA, RVH, Cushing's syndrome due to $\mathrm{CS}$ and $\mathrm{CD}, \mathrm{PCS}$, and $\mathrm{Ph}$ among the 1,020 hypertensive patients were $6.0 \%, 0.5 \%, 1.0 \%$, $1.0 \%$ and $0.6 \%$, respectively (Table 3 ). 
Table 6. Follow-Up Studies on Blood Pressure 1 Year after Surgical Treatment in Patients with APA, UMN, UAH, IHA, RVH, CS, PCS, CD and Ph

\begin{tabular}{|c|c|c|c|c|c|c|}
\hline & \multicolumn{3}{|c|}{ SBP } & \multicolumn{3}{|c|}{ DBP } \\
\hline & $\begin{array}{l}\text { Before treatment } \\
(\mathrm{mmHg})\end{array}$ & $\begin{array}{l}\text { After treatment } \\
\quad(\mathrm{mmHg})\end{array}$ & $p$ value & $\begin{array}{l}\text { Before treatment } \\
\quad(\mathrm{mmHg})\end{array}$ & $\begin{array}{l}\text { After treatment } \\
\quad(\mathrm{mmHg})\end{array}$ & $p$ value \\
\hline $\operatorname{APA}(n=45)$ & $175 \pm 3$ & $138 \pm 3$ & $<0.001$ & $97 \pm 2$ & $83 \pm 2$ & $<0.001$ \\
\hline $\mathrm{UMN}(n=3)$ & $163 \pm 3$ & $132 \pm 4$ & $<0.001$ & $95 \pm 2$ & $79 \pm 1$ & 0.015 \\
\hline UAH $(n=1)$ & 148 & 128 & & 100 & 80 & \\
\hline IHA $(n=1)$ & 160 & 170 & & 96 & 100 & \\
\hline RVH $(n=5)$ & $177 \pm 8$ & $138 \pm 15$ & 0.018 & $96 \pm 7$ & $86 \pm 3$ & NS \\
\hline $\operatorname{CS}(n=6)$ & $176 \pm 7$ & $126 \pm 4$ & $<0.001$ & $96 \pm 5$ & $73 \pm 2$ & 0.003 \\
\hline $\operatorname{PCS}(n=5)$ & $161 \pm 4$ & $132 \pm 3$ & 0.011 & $91 \pm 3$ & $74 \pm 4$ & NS \\
\hline $\mathrm{CD}(n=5)$ & $155 \pm 5$ & $119 \pm 5$ & 0.013 & $95 \pm 7$ & $69 \pm 5$ & 0.016 \\
\hline $\mathrm{Ph}(n=6)$ & $165 \pm 7$ & $115 \pm 5$ & 0.001 & $97 \pm 7$ & $71 \pm 4$ & 0.011 \\
\hline
\end{tabular}

Numbers of patient treated by unilateral adrenalectomy, transsphenoidal pituitary adenomectomy and percutaneous transluminal angioplasty are shown in parentheses. All data are shown as the means \pm SEM. Student's $t$-test was used to compare changes in each parameter between the preoperative and postoperative stages. Values of $p<0.05$ were considered to indicate statistical significance. NS, not significant; SBP, systolic blood pressure; DBP, diastolic blood pressure; APA, aldosterone-producing adenoma; UMN, unilateral multiple adrenocortical micronodules; UAH, unilateral adrenal hyperplasia; IHA, idiopathic hyperaldosteronism; RVH, renovascular hypertension; CS, Cushing's syndrome due to adrenocortical adenoma; PCS, preclinical Cushing's syndrome; CD, Cushing's disease; $\mathrm{Ph}$, pheochromocytoma.

Table 7. Incidence of Typical and Specific Symptoms and Signs among Subjects with Secondary Hypertension

\begin{tabular}{lcccc}
\hline & $\begin{array}{c}\text { Number of } \\
\text { patients }\end{array}$ & $\begin{array}{c}\text { Number of patients } \\
<35 \text { years }\end{array}$ & $\begin{array}{c}\text { Number of patients } \\
\text { with severe hypertension }\end{array}$ & Percentage of typical and specific symptoms and signs \\
\hline PA & 61 & 2 & 15 & Hypokalemia 24.6\% $(n=15)$ \\
RVH & 5 & 1 & 2 & Vascular murmur 0\% $(n=0)$ \\
CS & 6 & 1 & 3 & Cushingoid 66.7\% $(n=4)$ \\
PCS & 10 & 0 & 2 & Cushingoid 60\% $(n=3)$ \\
CD & 5 & 1 & 0 & History of palpitation $16.7 \%(n=1)$ \\
Ph & 6 & 0 & 2 & \\
\hline
\end{tabular}

Numbers of patients demonstrating clinical characteristics of secondary hypertension are shown in parentheses. PA, primary aldosteronism; RVH, renovascular hypertension; CS, Cushing's syndrome due to adrenocortical adenoma; PCS, preclinical Cushing's syndrome; $\mathrm{CD}$, Cushing's disease; $\mathrm{Ph}$, pheochromocytoma.

\section{Sensitivity and Specificity of Primary and Secondary Screening Tests}

As shown in Table 4, the primary and secondary screening tests, excluding US and CT, were sensitive and specific for differentiating $\mathrm{SH}$ from $\mathrm{EH}$.

\section{Outcome of Surgical Treatment of Secondary Hyper- tension}

At 1 year after unilateral adrenalectomy, 36 of 50 patients with PA became normotensive without any antihypertensive agents, and hypertension in 13 patients was partially controlled by lower doses and fewer antihypertensive drugs than before adrenalectomy. In 1 patient with IHA, hypertension was not improved after unilateral adrenalectomy.
We performed PTA for 5 patients with RVH after obtaining informed consent. At 1 year after PTA, hypertension was cured in 3 patients with RVH caused by fibromuscular renal artery stenosis and improved in 2 by atherosclerotic renal artery stenosis. At 1 year after unilateral adrenalectomy, hypertension was cured in 5 of 6 patients with CS and 4 of 5 patients with PCS, and improved in 1 patient each with CS and PCS. Hypertension in all 5 patients with CD was cured after transsphenoidal pituitary adenectomy.

At 1 year after unilateral adrenalectomy, hypertension was cured in 5 of 6 patients with $\mathrm{Ph}$, and improved in the remaining patient (Table 5 and 6). 


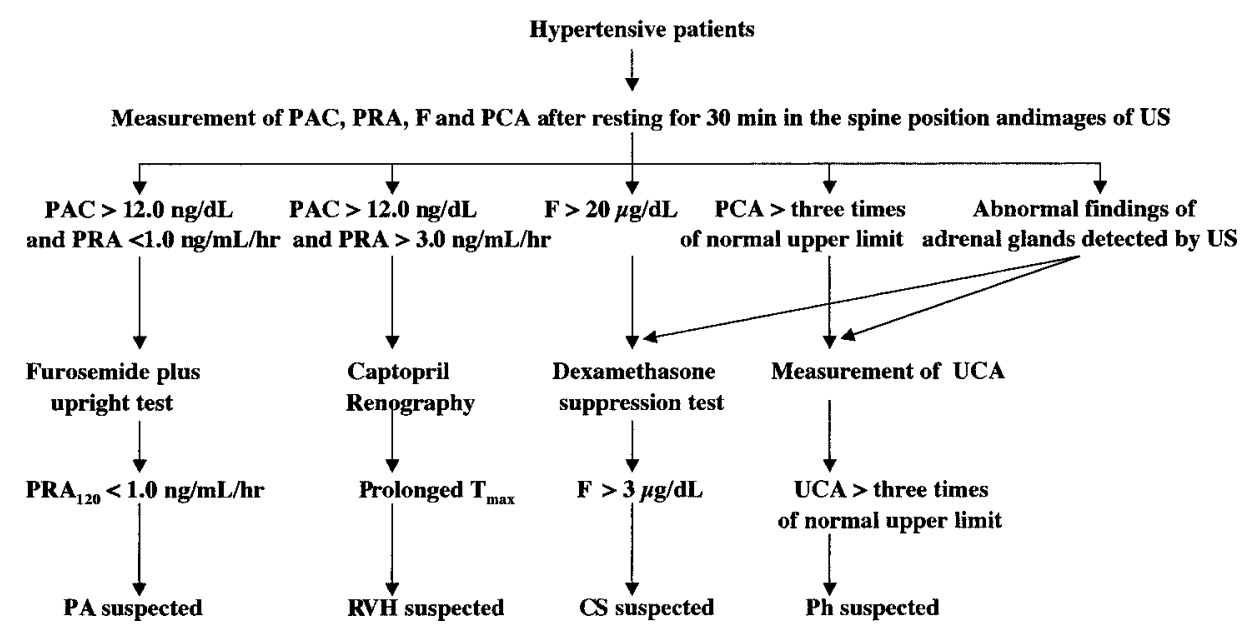

Fig. 5. Diagram for differentiating each disorder of secondary hypertension. PAC, plasma aldosterone concentration; PRA, plasma renin activity; F, serum cortisol concentration; PCA, plasma catecholamine concentration; US, ultrasonography; UCA, 24-h urine catecholamines; PRA120, PRA 120 min after intravenous administration of $40 \mathrm{mg}$ furosemide and standing upright; $\mathrm{T}_{\max }$, the time to maximal activity; PA, primary aldosteronism; RVH, renovascular hypertension; CS, Cushing's syndrome; Ph, pheochromocytoma.

\section{Presence of Specific Symptoms and Signs Suggest- ing the Existence of Secondary Hypertension}

Five patients with $\mathrm{SH}$ were younger than 35 years old. Severe hypertension (systolic blood pressure $>180 \mathrm{mmHg}$ and/or diastolic blood pressure $>110 \mathrm{mmHg}$ ) was observed in $25.8 \%$ of 93 patients with SH. Hypokalemia (serum potassium $<3.3 \mathrm{mEq} / \mathrm{l}$ ) was found in $24.6 \%$ of 61 patients with PA. Vascular murmur of the abdomen was not audible in any of the 6 patients with RVH. Cushingoid features such as central obesity, moon face and purplish stria were observed in 4 patients with CS and in 3 with CD. Only 1 patient with $\mathrm{Ph}$ had a history of palpitation, which is well known to be one of the typical symptoms of $\mathrm{Ph}$ (Table 7).

\section{Discussion}

The present investigations clearly demonstrated that the prevalence of $\mathrm{SH}$, including endocrine and renovascular hypertension, was $9.1 \%$ among 1,020 hypertensive patients in our clinic. Our data also showed that the blood pressure was completely normalized to less than $140 / 90 \mathrm{mmHg}$ in 58 patients and improved in 18 of 77 patients with SH who were treated by surgical procedures including unilateral adrenalectomy, PTA and transsphenoidal pituitary adenectomy. Therefore, the differential diagnosis of curable $\mathrm{SH}$ from $\mathrm{EH}$ is very important.

Guidelines for the management of hypertension have recommended performing further examinations such as hormonal tests and renography when clinical features suggesting $\mathrm{SH}$ are observed among hypertensives $(2,19)$. It has also been reported that patients with PA do not always show hypokalemia (20), and that only $33 \%$ of patients with Ph pres- ent two or more symptoms of the classical triad, such as headache, palpitation and diaphoresis (21). Retrospective investigations have reported that central obesity and moon face are common in patients with Cushing's syndrome (22), while it is difficult to differentiate hypertensive patients complicated with obesity from patients with Cushing's syndrome, especially among females. In the present study there were few patients showing typical symptoms and signs of SH. Thus, we would like to emphasize that all hypertensive patients should be carefully examined for the etiology of hypertension at the first visit to a general outpatient clinic.

The sensitivity and specificity of the primary screening tests, i.e., PAC, PRA, $F$ and PCA measurement, were high enough to distinguish $\mathrm{SH}$ from EH. As shown in the Methods, we carefully measured PAC, PRA, $F$ and PCA after the patient had rested in a supine position for $30 \mathrm{~min}$, and before initiating treatment with antihypertensive drugs, since physical and mental stress, posture and antihypertensive drugs (23-25) other than budralazine alter PAC, PRA, F, and PCA. It is possible that this thorough methodology played a role in our obtaining a higher prevalence of $\mathrm{SH}$ than that previously reported $(3-5,12)$.

The aldosterone renin ratio (ARR) has been generally accepted as an efficient screening tool for diagnosing PA since it was first reported by Hiramatsu et al. (26). However, the sensitivity and specificity of ARR have varied in previous reports (27-29), suggesting that while ARR may be a simple method for screening PA among all hypertensive subjects, the ratio is not sensitive enough to differentiate PA from EH. Kaplan cautioned that values might be better expressed as subnormal PRA and raised aldosterone rather than in the form of a ratio (30). Thus, we used the criteria PRA $<1.0 \mathrm{ng} / \mathrm{ml} / \mathrm{h}$ and PAC $>12.0 \mathrm{ng} / \mathrm{dl}$ for the diagnosis of hy- 
poreninemic hyperaldosteronemia.

As a primary screening test for SH, US is not sensitive in detecting an adrenal mass because of its limitation in detecting small lesions (Table 4). However, our data suggested that US is quite simple and beneficial for the detection of adrenal masses inducing $\mathrm{SH}$, even in patients with hypertension showing normal values of $F$ and PCA (Table 2).

As secondary screening tests, the furosemide plus upright test, dexamethasone suppression test, captopril renography, and UCA measurement proved to be sensitive and specific enough to differentiate endocrine hypertension from $\mathrm{EH}$ (Table 4). These secondary screening tests increased the accuracy and efficacy of screening for SH. On the other hand, the sensitivity of an adrenal CT scan was not adequate, especially for detecting APA. In this series, 45 patients with adrenal tumors detected by CT had already been identified by primary screening tests. However, CT failed to demonstrate APA in 22 patients who were suspected of PA based on PAC and PRA measurements as well as the furosemide plus upright test. Therefore, CT seems to be inadequate as a screening test for $\mathrm{SH}$ in some cases. We would like to propose a simple screening scheme for $\mathrm{SH}$, as shown in Fig. 5.

We have already reported the incidence of PA in these 1,020 patients in our previous report; however, we diagnosed 6 more patients with PA in this population after submitting the previous report (11). The incidence of PA from the present study is quite similar to that recently reported $(6-10)$. We recommend always checking PAC and PRA when caring for hypertensive patients, since PA is a main cause of $\mathrm{SH}$.

As shown in Table 3, the prevalence of pheochromocytoma and Cushing's syndrome has been reported to be low $(<0.6 \%$ each) among all hypertensive patients $(3-5,12)$. However, the prevalence of CS in the present study was 2-10 times higher and that of $\mathrm{Ph} 2-6$ times higher than those previously reported $(3-5,12)$. A noteworthy finding is that 10 patients with PCS manifested only hypertension. Therefore, it is also necessary to differentiate CS, PCS and $\mathrm{Ph}$ from $\mathrm{EH}$ using our screening tests.

On the other hand, the prevalence of RVH was lower than that reported previously $(4,5,12)$, and the reason for this difference is unknown. Atherosclerotic renal artery stenosis was found in $41 \%$ of patients starting dialysis (31), and $7-19 \%$ of patients were referred for coronary angioplasty $(32,33)$. In the present study, we examined patients who were first diagnosed with hypertension at our general outpatient clinic and excluded patients with renal failure, heart failure, acute-phase coronary heart disease, or acute-phase cerebrovascular disease from our study. Therefore, most of the patients analyzed were not suspected of having severe atherosclerotic complications. The incidence of RVH caused by atherosclerotic changes of the renal arteries was probably lower in our study than in past reports.

PTA was first recommended as one of the best treatments for patients with $\mathrm{RVH}(34,35)$, especially for those with fibromuscular renal artery stenosis. Moreover, in patients with atherosclerotic renal artery stenosis, the cure rate after PTA was $8-10 \%$ and improvement in BP was observed in 40-50\% patients (36). Therefore, we attempted PTA for treatment of RVH to avoid long-term use of antihypertensive agents.

In conclusion, the present study was performed in a single facility of Yokohama Rosai Hospital and was the first investigation of the prevalence of $\mathrm{SH}$ in Japan. Our present prospective study on the prevalence of SH among 1,020 hypertensive patients showed a high prevalence of PA, Cushing's syndrome including CS, PCS and CD, and Ph. Therefore, we propose that our sequential screening system (Fig. 5 ) is an effective method to differentiate $\mathrm{SH}$ from $\mathrm{EH}$. We recommend that physicians screen for $\mathrm{SH}$ before initiating treatment with antihypertensive agents, and treat patients with SH individually using appropriate therapies in order to avoid long-term use of antihypertensive drugs for maintaining QOL while preventing various cerebro-cardiovascular complications and reducing medical costs.

\section{References}

1. Wolf-Maier K, Cooper RS, Banegas JR, et al: Hypertension prevalence and blood pressure levels in 6 European countries, Canada, and the United States. JAMA 2003; 289: 2363-2369.

2. Japanese Society of Hypertension Guidelines Subcommittee for the Management of Hypertension: Guidelines for the management of hypertension for general practitioners. $\mathrm{Hy}$ pertens Res 2001; 24: 613-634.

3. Rudnick KV, Sackett DL, Hirst S, et al: Hypertension in a family practice. Can Med Assoc J 1977; 117: 492-497.

4. Danielson M, Dammström B: The prevalence of secondary and curable hypertension. Acta Med Scand 1981; 209: 451-455.

5. Sinclair AM, Isles CG, Brown I, et al: Secondary hypertension in a blood pressure clinic. Arch Intern Med 1987; 147: 1289-1293.

6. Gordon RD, Stowasser M, Tunny TJ, et al: High incidence of primary aldosteronism in 199 patients referred with hypertension. Clin Exp Pharmacol Physiol 1994; 21: 315-318.

7. Komiya I, Yamada T, Takasu N, et al: An abnormal sodium metabolism in Japanese patients with essential hypertension, judged by serum sodium distribution, renal function and the renin-aldosterone system. J Hypertens 1997; 15: $65-72$.

8. Lim PO, Rodgers $\mathrm{P}$, Cardale $\mathrm{K}$, et al: Potentially high prevalence of primary aldosteronism in a primary-care population. Lancet 1999; 353: 40.

9. Loh K, Koay E, Khaw M, et al: Prevalence of primary aldosteronism among Asian hypertensive patients in Singapore. J Clin Endocrinol Metab 2000; 85: 2854-2859.

10. Rossi E, Regolisti G, Negro A, et al: High prevalence of primary aldosteronism using postcaptopril plasma aldosterone to renin ratio as a screening test among Italian hypertensives. Am J Hypertens 2002; 15: 896-902.

11. Nishikawa T, Omura M: Clinical characteristics of primary aldosteronism: its prevalence and comparative studies on 
various causes of primary aldosteronism in Yokohama Rosai Hospital. Biomed Pharmacother 2000; 54 (Suppl 1):, $83 \mathrm{~s}-85 \mathrm{~s}$.

12. Anderson GH Jr, Blakeman N, Streeten DHP: The effect of age on prevalence of secondary forms of hypertension in 4429 consecutively referred patients. J Hypertens $1994 ; \mathbf{1 2}$ : 609-615.

13. Mikami K, Nishikawa T, Tamura $\mathrm{Y}$, et al: Inter-relationship of sympathetic nervous system and renin-angiotensinaldosterone system in three renin subgroups of borderline and persistent essential hypertension. Clin Exp Hypertens 1981; 3: 1091-1107.

14. Helin KH, Tikkanen I, von Knorring JE, et al: Screening for renovascular hypertension in a population with relatively low prevalence. J Hypertens 1998; 16: 1523-1529.

15. Nugent CA, Nicholis T, Tyler FH: Diagnosis of Cushing's syndrome: single dose dexamethasone suppression test. Arch Intern Med 1965; 116: 172-176.

16. Naomi S, Iwaoka T, Umeda T, et al: Clinical evaluation of captopril screening test for primary aldosteronism. Jpn Heart J 1985; 26: 549-556.

17. Omura M, Sasano H, Fujiwara T, et al: Unique cases of unilateral hyperaldosteronemia due to multiple adrenocortical micronodules, which can only be detected by selected adrenal venous sampling. Metabolism 2002; 51: 350-355.

18. Ross EJ: Conn's syndrome due to adrenal hyperplasia with hypertrophy of zona glomerulosa, relieved by unilateral adrenalectomy. Am J Med 1965; 39: 994-1001.

19. Guideline Committee: 2003 European Society of Hypertension-European Society of Cardiology guidelines for the management of arterial hypertension. J Hypertens 2003; 21: 1011-1053.

20. Fardella CE, Mosso L: Author's response; prevalence of primary aldosteronism in unselected hypertensive populations: screening and definitive diagnosis. J Clin Endocrinol Metab 2001; 86: 4003-4004.

21. Loh KC, Shlossberg AH, Abbott EC, et al: Phaeochromocytoma: a ten-year survey. QJM 1997; 90: 51-60.

22. Boscaro M, Barzon L, Fallo F, et al: Cushing's syndrome. Lancet 2001; 357: 783-791.

23. Mulatero P, Rabbia F, Milan A, et al: Drug effects on aldosterone/plasma renin activity ratio in primary aldosteronism. Hypertension 2002; 40: 897-902.

24. Seifarth C, Trenkel S, Schobel H, et al: Influence of antihypertensive medication on aldosterone and renin concentration in the differential diagnosis of essential hypertension and primary aldosteronism. Clin Endocrinol (Oxf) 2002; 57: 457-465.

25. Montori VM, Schwartz GL, Chapman AB, et al: Validity of the aldosterone-renin ratio used to screen for primary aldosteronism. Mayo Clin Proc 2001: 76: 877-882.

26. Hiramatsu K, Yamada T, Yukimura Y, et al: A screening test to identify aldosterone-producing adenoma by measurement plasma renin activity. Arch Intern Med 1981; 141: 1589-1593.

27. Fardella CE, Mosso L, Gómez-Sánchez C, et al: Primary aldosteronism in essential hypertensives: prevalence, biochemical profile, and molecular biology. J Clin Endocrinol Metab 2000; 85: 1863-1867.

28. Hirohara D, Nomura K, Okamoto T, et al: Performance of the basal aldosterone to renin ratio and of the renin stimulation test by furosemide and upright posture in screening for aldosterone-producing adenoma in low renin hypertensives. J Clin Endocrinol Metab 2001; 86: 4292-4298.

29. Montori VM, Young WF Jr: Use of plasma aldosterone concentration-to-plasma renin activity ratio as a screening test for primary aldosteronism: a systematic review of the literature. Endocrinol Metab Clin North Am 2002; 31: 619-632.

30. Kaplan NM: Caution over the current epidemic of primary aldosteronism. Lancet 2001; 357: 953-954.

31. Van Ampting JM, Penne EL, Beek FJ, et al: Prevalence of atherosclerotic renal artery stenosis in patients starting dialysis. Nephrol Dial Transplant 2003; 18: 1147-1151.

32. Yamashita $\mathrm{T}$, Ito $\mathrm{F}$, Iwakiri N, et al: Prevalence and predictors of renal artery stenosis in patients undergoing cardiac catheterization. Hypertens Res 2002; 25: 553-557.

33. Khosla S, Kunjummen B, Manda R, et al: Prevalence of renal artery stenosis requiring revascularization in patients initially referred for coronary angiography. Cathet Cardiovasc Intervent 2003; 58: 400-403.

34. Martinez-Amenos A, Rama H, Sarrias X, et al: Percutaneous transluminal angioplasty in the treatment of renovascular hypertension. J Hum Hypertens 1991; 5: 97-100.

35. Xue F, Bettmann MA, Langdon DR, et al: Outcome and cost comparison of percutaneous transluminal renal angioplasty, renal arterial stent placement, and renal arterial bypass grafting. Radiology 1999; 212: 378-384.

36. Morganti A: Angioplasty of the renal artery: antihypertensive and renal effects. J Nephrol 2000; 13 (Suppl): S28S33. 